\title{
DEGRADABILIDAD RUMINAL DE LA PLANTA DE SORGO NEGRO FORRAJERO (Sorghum almum) EN DIFERENTES ETAPAS DE CRECIMIENTO 1
}

\author{
Carlos Boschini ${ }^{2}$, Ana Lorena Amador ${ }^{2}$
}

\begin{abstract}
RESUMEN
Degradabilidad ruminal de la planta de sorgo negro forrajero (Sorghum almum) en diferentes etapas de crecimiento. El trabajo se llevó a cabo con el propósito de conocer la degradabilidad ruminal de la materia seca del sorgo negro forrajero, cultivado en una zona alta de Costa Rica. Se tomaron muestras en diferentes etapas de crecimiento de la planta. El estudio se realizó en la Estación Experimental "Alfredo Volio Mata" de la Universidad de Costa Rica. El cultivo fue sembrado con $18 \mathrm{~kg} / \mathrm{ha}$ de semilla ( $88 \%$ semilla pura germinable), distribuida a chorro continuo en surcos distanciados a $70 \mathrm{~cm}$. Se fertilizó con $244 \mathrm{~kg}$ de la fórmula comercial 10-30-10 $\left(\mathrm{N}_{2}, \mathrm{P}_{2} \mathrm{O}_{5}, \mathrm{~K}_{2} \mathrm{O}\right)$ y a las seis semanas se aplicaron $45,5 \mathrm{~kg} / \mathrm{ha}$ de nitrógeno. El primer muestreo se realizó a los 24 días de edad y los siguientes, cada 14 días, hasta los 150 días. Se determinó la degradabilidad potencial de la materia seca en las hojas, tallos, panojas. La degradabilidad potencial de la planta entera disminuyó de 90 a $75 \%$ conforme aumentó la edad durante los primeros 94 días. En el tallo, la fracción soluble disminuyó de 32 a 23\%, mientras que la hoja bajó de 30 a 16\%. La fracción degradable en el tallo disminuyó de 57 a $46 \%$ con el desarrollo de la planta, en tanto que en la hoja aumentó de 56 a 69\%. La velocidad de degradación del tallo fue de $4,7 \% / \mathrm{h}$ a los 52 días y en la hoja de $8,6 \% / \mathrm{h}$. De 66 a 94 días fue de $2,6 \% / \mathrm{h}$ en el tallo y en la hoja de $5,4 \% / \mathrm{h}, 3,3 \% / \mathrm{h}$ y $3,8 \% / \mathrm{h}$ a $\operatorname{los} 66,80$ y 94 días, respectivamente.
\end{abstract}

\begin{abstract}
Ruminal degradability of black sorghum (Sorghum almum) fodder fiber in different growth stages. The purpose of this study was to determine the ruminal degradability of dry material of black sorghum fodder, cultivated in the highlands of Costa Rica. Samples were taken at different growth periods of the plant. The study was conducted at the Alfredo Volio Mata Experimental Station of the University of Costa Rica. The crop was planted with 18 $\mathrm{kg} / \mathrm{ha}$ of seed (88\% pure germinal seed), distributed continuously in furrows separated by $70 \mathrm{~cm}$. It was fertilized with $244 \mathrm{~kg}$ of $10-30-10\left(\mathrm{~N}_{2}, \mathrm{P}_{2} \mathrm{O}_{5}, \mathrm{~K}_{2} \mathrm{O}\right)$ and after 6 weeks, $45.5 \mathrm{~kg} / \mathrm{ha}$ of nitrogen was applied. The first sample was taken after 24 days and then every 14 days for a total of 150 days. The potential degradability of the dry material was determined for the leaves, stems and panicles. The potential degradability of the whole plant diminished from $90 \%$ to $75 \%$ as the plant grew during the first 94 days. In the stem, the soluble part diminished from $32 \%$ to $23 \%$, while that of the leaves was reduced from $30 \%$ to $16 \%$. The degradable portion in the stem was reduced from $57 \%$ to $46 \%$ as the plant developed, while that of the leaves increased from 56\% to $69 \%$. The degradation rate in the stems was $4.7 \% / \mathrm{h}$ after 52 days and in the leaves it was $8.6 \% / \mathrm{h}$. Between the 66th and the 94 th days it was $2.6 \% / \mathrm{h}$ in the stems. In the leaves it was $5.4 \% / \mathrm{h}, 3.3 \% / \mathrm{h}$ and $3.8 \% / \mathrm{h}$ on the 66 th, 80 th and 94 th day, respectively.
\end{abstract}

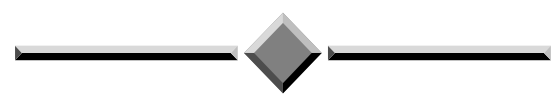

\section{INTRODUCCIÓN}

La producción de sorgo negro para la alimentación forrajera del ganado lechero se ha extendido en Costa Rica con rendimientos de materia seca de seis $\mathrm{t} / \mathrm{ha} /$ corte en la zona alta (Amador y Boschini 2000) y de 10 t/ha/corte en la zona baja (Corrales 1986). Se cultiva para la producción de forraje de corte y suministrarlo picado en fresco o conservado mediante ensilaje (Villegas 1990). Es un material que se conserva fácilmente debido a su alto contenido de sustancias solubles, siguiendo los procedimientos de elaboración de ensilaje adecuados. La disponibilidad de este material permite una mejor alimentación del ganado en los periodos secos del trópico y posee una gran habilidad para recuperarse de la sequía (Owen y Moline 1970). Se adapta bien desde el nivel del mar hasta los 1900-2000 msnm (Huges 1978, Villegas 1990) y temperaturas superiores a los $13^{\circ} \mathrm{C}$. Hernández (1989) propone una edad de cosecha de 55 días de rebrote en Tilarán $(560 \mathrm{msnm})$,

1 Recibido para publicación el 19 de octubre de 1999. Proyecto No. 737-97-006 inscrito en la Vicerrectoría de Investigación, Universidad de Costa Rica.

2 Estación Experimental Alfredo Volio Mata. Facultad de Agronomía. Universidad de Costa Rica. E-mail: boschini@cariari.ucr.ac.cr. Tel: (506) 279-5840, 279-5850. 
mientras que Hernández (1986) recomienda una edad de 63 días en el Alto de Ochomogo (Estación Experimental Alfredo Volio Mata), Costa Rica. El contenido nutricional varía con la edad y la altitud, con valores de $12,5 \%$ de proteína cruda a los 45 días y de $9,5 \%$ a los 55 días en zonas bajas (Villegas 1990) y de 22 a $18 \%$ en ese mismo periodo en zonas altas y un contenido de $14 \%$ a los 80 días de rebrote (Amador y Boschini 2000). En zonas bajas y cálidas, se ha encontrado digestibilidades in vitro de la materia seca de $65 \%$ a los 45 días y de $60 \%$ a los 60 días de rebrote. Digestibilidad in vitro de $80 \%$ a los 49 días y de $71 \%$ a los días fueron encontrados por Hernández (1989). El contenido de fibra neutro detergente varía de 56 y $63 \%$ en la planta entera, de 56 y $62 \%$ en la hoja y de 58 y $64 \%$ en el tallo en las edades de 52 y 66 días (Amador y Boschini 2000).

El presente trabajo se llevó a cabo con el propósito de conocer la degradabilidad ruminal de la materia seca del sorgo negro forrajero en las diferentes etapas de crecimiento de la planta.

\section{MATERIALES Y MÉTODOS}

El estudio se llevó a cabo en la Estación Experimental Alfredo Volio Mata de la Universidad de Costa Rica; ubicada en El Alto de Ochomogo, Cartago; a $1542 \mathrm{msnm}$, con una precipitación media anual de $2050 \mathrm{~mm}$, distribuida de mayo a noviembre. La temperatura media es de $19,5^{\circ} \mathrm{C}$ y la humedad relativa media es de $84 \%$. El suelo es de origen volcánico, clasificado como Typic Distrandepts (Vásquez 1982), que se caracteriza por una profundidad media, con buen drenaje natural y fertilidad media. El terreno empleado tenía un pH de 6. La zona se tipifica ecológicamente como bosque húmedo montano bajo (Tosi 1970, citado por Vásquez 1982).

Una hectárea de terreno fue arada, rastreada y surcada a una distancia de $70 \mathrm{~cm}$. La siembra del ensayo se realizó la cuarta semana de mayo de 1999. Se emplearon $18 \mathrm{~kg} / \mathrm{ha}$ de semilla de sorgo negro forrajero (88\% semilla pura germinable), en una mezcla con tierra seca fina en una proporción de $1 \mathrm{a} 1 \mathrm{v} / \mathrm{v}$, distribuida a chorro continuo. Se fertilizó con $368 \mathrm{~kg}$ de formula 10-30-10 $\left(\mathrm{N}_{2}, \mathrm{P}_{2} \mathrm{O}_{5}, \mathrm{~K}_{2} \mathrm{O}\right)$. A los 24 días de edad se realizó el primer muestreo de plantas y los siguientes se efectuaron cada 14 días hasta completar 150 días de edad. A los 40 días de la siembra se aplicaron $45,5 \mathrm{~kg}$ de nitrógeno en forma de nitrato de amonio. Los sitios de muestreos se realizaron al azar, recolectando $10 \mathrm{~m} \mathrm{li-}$ neales en los surcos previamente seleccionados. Las plantas fueron cortadas a tres $\mathrm{cm}$ sobre el nivel del sue- lo. Los tallos, hojas y panojas fueron separados, pesados y secados en un horno a $60^{\circ} \mathrm{C}$ durante $48 \mathrm{~h}$, hasta alcanzar peso constante. Posteriormente se molieron a $2 \mathrm{~mm}$. La degradación ruminal in situ de la materia seca de las muestras se efectuó siguiendo la técnica descrita por Orskov (1984) con bolsas de nylon. Se usaron bolsas de $12 \times 6 \mathrm{~cm}$ con un poro de $45 \mathrm{~mm}$, conteniendo cada una cinco gramos de muestra secada a $105^{\circ} \mathrm{C}$. Las bolsas de nylon fueron suspendidas en el rumen de dos novillos fistulados ruminalmente, que fueron mantenidos con una dieta a base de pasto Estrella Africana (Cynodon nlemfluensis). Las bolsas, en dos repeticiones, fueron introducidas consecutivamente en el rumen y mantenidas por seis, 12, 24, 48 y 72 horas. La desaparición a cero horas de incubación fue evaluada por inmersión de las bolsas en agua a $40^{\circ} \mathrm{C}$ por una hora. Al completar cada periodo de incubación, las bolsas fueron removidas del rumen juntas e inmediatamente lavadas en abundante agua fría. La materia seca residual fue medida, después de que las bolsas fueron secadas a $105^{\circ} \mathrm{C}$. La cinética de la degradación fue determinada con el procedimiento descrito por Orskov y McDonald (1979). Los datos fueron ajustados a la ecuación exponencial $\mathrm{P}=\mathrm{a}+\mathrm{b}\left(1-\mathrm{e}^{-\mathrm{ct}}\right)$, donde $\mathrm{P}=$ degradación después de $t$ horas, $a=$ fracción solubre, $b=$ fracción degradable, e=base de los logaritmos neperianos, $\mathrm{c}=$ tasa de degradación por hora y $\mathrm{t}=$ tiempo de incubación ruminal: las constante fueron calculadas por un programa de cuadrados mínimos iterativo, usando el algoritmo de Marquardt.

\section{RESULTADOS}

Se estimó la degradabilidad potencial de la materia seca en las hojas, tallos y panojas del sorgo negro, así como los componentes fraccionados: parte soluble, parte degradable y la velocidad de degradación de esta fracción insoluble. Los valores de las fracciones de la planta entera fueron estimados, ponderando el valor de cada fracción en la parte de la planta con la aportación de materia seca respectiva en la planta entera. En el Cuadro 1 se presentan los resultados obtenidos para el tallo, la hoja, la panoja y la planta entera. Se observa que la degradabilidad potencial de la planta entera fue disminuyendo de 90 a $75 \%$ conforme aumentó la edad durante los primeros 94 días, en estados de prefloración. En este periodo, la fracción soluble del tallo disminuyó de $32 \%$ a $23 \%$, mientras que en la hoja bajó de $30 \%$ a $16 \%$. La fracción insoluble en el tallo disminuyó de $57 \%$ a $46 \%$ con el desarrollo de la planta, en tanto que en la hoja aumentó de $56 \%$ a $69 \%$. La velocidad de degradación del tallo fue de $4,7 \% / \mathrm{h}$ a los 52 días y en la hoja de $8,6 \% / \mathrm{h}$ y en la hoja de $5,4 \% / \mathrm{h}$ a $\operatorname{los} 66$ días y de 3,3 a 3,8 \%/h a los 80 y 94 días. La Figura 1 
Cuadro 1. Valores de degradabilidad ruminal de la materia seca en la planta de sorgo negro forrajero, en sus diferentes partes y edades de crecimiento. Estación Experimental Alfredo Volio Mata, El Alto Ochomogo, Costa Rica 1999.

\begin{tabular}{|c|c|c|c|c|c|c|c|}
\hline $\begin{array}{l}\text { Edad en } \\
\text { días }\end{array}$ & $\begin{array}{l}\text { Parte de } \\
\text { la planta }\end{array}$ & $\begin{array}{l}\text { Fracción } \\
\text { soluble (\%) }\end{array}$ & $\begin{array}{l}\text { Fracción } \\
\text { degradable (\%) }\end{array}$ & $\begin{array}{c}\text { Tasa de } \\
\text { degradación }(\% / h)\end{array}$ & $\begin{array}{c}\text { Degradabilidad } \\
\text { a } 24 \text { horas }(\%)\end{array}$ & $\begin{array}{c}\text { Degradabilidad } \\
\text { potencial }(\%)\end{array}$ & $\begin{array}{c}\text { Distribución } \\
\text { materia seca (\%) }\end{array}$ \\
\hline \multirow[t]{3}{*}{52} & Tallo & 32,03 & 57,19 & 0,047003 & 50,54 & 89,22 & 28,78 \\
\hline & Hoja & 30,14 & 56,62 & 0,086426 & 37,25 & 86,76 & 71,22 \\
\hline & Entera & 30,68 & 56,78 & 0,075081 & 40,05 & 87,47 & \\
\hline \multirow[t]{3}{*}{66} & Tallo & 26,84 & 64,31 & 0,026280 & 61,07 & 91,15 & 59,32 \\
\hline & Hoja & 20,82 & 67,83 & 0,054793 & 39,03 & 88,65 & 40,68 \\
\hline & Entera & 24,39 & 65,74 & 0,037879 & 52,10 & 90,13 & \\
\hline \multirow[t]{3}{*}{80} & Tallo & 19,86 & 49,97 & 0,027858 & 45,47 & 69,83 & 53,27 \\
\hline & Hoja & 16,67 & 71,08 & 0,033017 & 48,85 & 87,75 & 46,73 \\
\hline & Entera & 18,37 & 59,84 & 0,030269 & 47,31 & 78,20 & \\
\hline \multirow[t]{3}{*}{94} & Tallo & 23,30 & 46,03 & 0,024792 & 48,69 & 69,33 & 66,67 \\
\hline & Hoja & 16,83 & 69,33 & 0,038172 & 44,57 & 86,16 & 33,33 \\
\hline & Entera & 21,14 & 53,80 & 0,029252 & 47,80 & 74,94 & \\
\hline \multirow[t]{3}{*}{108} & Tallo & 24,34 & 60,40 & 0,016250 & 65,23 & 84,74 & 71,50 \\
\hline & Hoja & 19,28 & 57,95 & 0,050023 & 36,72 & 77,23 & \\
\hline & Entera & 22,90 & 59,70 & 0,025874 & 54,98 & 82,60 & 28,50 \\
\hline \multirow[t]{4}{*}{122} & Tallo & 22,38 & 58,09 & 0,014883 & 63,02 & 80,47 & 66,12 \\
\hline & Hoja & 15,71 & 63,04 & 0,031223 & 45,51 & 78,75 & 21,98 \\
\hline & Panoja & 25,63 & 54,07 & 0,021735 & 57,72 & 79,70 & 11,90 \\
\hline & Entera & 21,30 & 58,70 & 0,019289 & 58,25 & 80,00 & \\
\hline \multirow[t]{4}{*}{136} & Tallo & 18,73 & 75,93 & 0,011262 & 76,68 & 94,66 & 67,82 \\
\hline & Hoja & 17,75 & 54,07 & 0,028133 & 45,27 & 71,82 & 12,60 \\
\hline & Panoja & 12,92 & 48,13 & 0,036775 & 32,83 & 61,05 & 19,58 \\
\hline & Entera & 17,47 & 67,73 & 0,018382 & 61,04 & 85,20 & \\
\hline \multirow[t]{4}{*}{150} & Tallo & 16,14 & 83,30 & 0,009034 & 83,20 & 99,44 & 62,80 \\
\hline & Hoja & 18,63 & 49,15 & 0,039864 & 37,51 & 67,78 & 13,62 \\
\hline & Panoja & 16,88 & 48,17 & 0,038135 & 36,17 & 65,05 & 23,58 \\
\hline & Entera & 16,65 & 70,37 & 0,020095 & 65,89 & 87,02 & \\
\hline
\end{tabular}

muestra la cinética de la degradación ruminal de las hojas y tallos a través de los tiempos de incubación ruminal durante los estados de prefloración. En el Cuadro 1 se observa que la contribución de materia seca proveniente del tallo va aumentando, en relación a la masa seca presente en la planta entera; mientras que la proporción de hojas en la planta va disminuyendo.

En el crecimiento de la planta después de los 94 días aparecen los estados de floración. La degradabilidad potencial de la planta entera se mantiene en valores cercanos a $80 \%$, aumentando la degradabilidad del tallo y decayendo la degradabilidad de la hoja conforme aumenta la edad. La panoja tiene una degradabilidad potencial de $79 \%$ a los 122 días, bajando a $65 \%$ a los 150 días. A partir de los 108 días, la velocidad de degradación de la planta entera disminuye de $2,5 \% / \mathrm{h}$ a $2,0 \% / \mathrm{h}$ a $\operatorname{los} 150$ días de edad. En tallos disminuye de $1,6 \% / \mathrm{h}$ a $0,9 \% / \mathrm{h}$ y en las hojas se mantiene en valores ligeramente superiores a 2,8\%/h. En la Figura 2 se presenta la cinética de la degradación ruminal de las hojas y los tallos y las panojas a través de los tiempos de incubación.

\section{DISCUSIÓN}

Las curvas de degradabilidad ruminal de la materia seca de las hojas, tallo y panojas del sorgo negro forra- jero, durante los diferentes estados de crecimiento, se presentaron en las Figuras 1 y 2. En los primeros estados de crecimientos (52 y 66 días), se observa que la degradabilidad potencial del tallo fue mayor que en la hoja. A los 80 y 94 días, la degradabilidad potencial de la hoja fue superior a la del tallo. Posteriormente, de los 108 días hasta los 150 días, los tallos mostraron de nuevo una degradabilidad potencial mayor que las hojas. La fracción soluble en el tallo fue mayor en el tallo durante los primeros 136 días de crecimiento, con una diferencia de 1 a $6 \%$ en sustancias solubles, siendo mayores en los estados iniciales de crecimiento. La fracción degradable fue similar en las edades de 52 y 66 días de crecimiento. A los 80 y 94 días, esta fracción insoluble aumentó en el tallo, mostrando diferencias con respecto a la hoja de 22\%. A partir de los 108 días, la fracción degradable en el tallo fue nuevamente superior en los tallos en relación a las hojas, con diferencias de 2 a $34 \%$ conforme aumentó la edad de la planta. Cabe indicar que la degradabilidad potencial de la planta entera de sorgo negro decae un $22 \%$ con el crecimiento, entre los 52 días y 94 días; posteriormente incrementa entre un 6 y 13\%. La aparición de la flor mejoró la degradabilidad potencial de la planta entera en los primeros estados y conforme progresa su maduración deteriora la degradabilidad del forraje. La degradabilidad potencial promedio de la materia seca de forrajes 

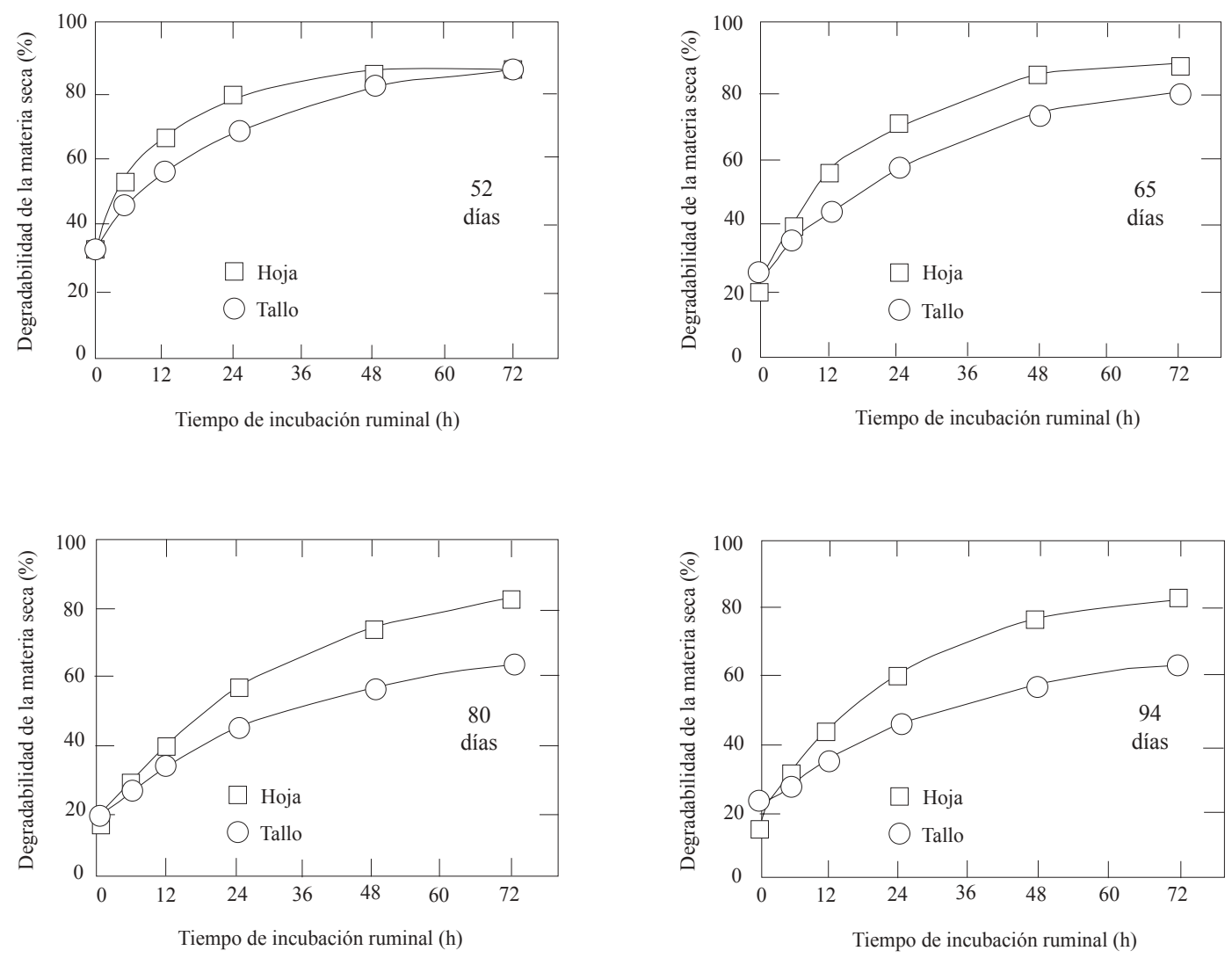

Figura 1. Degradabilidad ruminal de la materia seca de hojas y tallos de la planta de sorgo negro forrajero en estado de prefloración. Est. Exp. Alfredo Volio Mata, El Alto Ochomogo, Costa Rica 1999.

tropicales de corte como King Grass (Pennisetum hybridum) muestran valores de $70 \%$ y tasas de degradación de 3,3\%/h. Forrajes de piso como Estrella Africana (Cynodon nlemfluensis) presentan valores de 64\% de degradabilidad potencial y velocidades de degradación de 4,4\%/h (Abarca 1988, Medina 1988, Murillo 1990, Corado 1991, Moreno 1991, Jiménez 1995). El Kikuyo (Pennissetum clandestinum) tiene degradabilidades cercanas al $80 \%$ y tasas de casi 6\%/h (Zúñiga 1992). El sorgo negro forrajero muestra valores de degradabilidad superiores (8 a 20\%) al pasto King Grass y a la Estrella Africana en los primeros 80 días de crecimiento del sorgo. En los primeros 66 días de crecimiento, el sorgo muestra degradabilidades potenciales y tasas de degradación similares al Kikuyo. El maíz empleado como forraje, muestra valores de degradabilidad potencial y tasas de degradación superiores al sorgo negro en cerca de 10\% (Boschini y Amador 2001). Jiménez (1995) evaluando la cinética de degradación del sorgo negro reportó valores similares a los hallados en el presente estudio.

\section{CONCLUSIONES Y RECOMENDACIONES}

Se determinó la forma dinámica en que se lleva a cabo la degradación ruminal del sorgo negro forrajero, cosechado en las diferentes etapas de crecimiento de la planta. Conforme aumenta la edad del forraje, disminuye la fracción soluble de los tallos y de las hojas y aumenta la fracción degradable de la planta. Consecuentemente, la velocidad de degradación ruminal de esta última fracción disminuyó conforme aumentó la edad de crecimiento. El tallo mostró una mayor degradabilidad potencial que las hojas, desde edades tempranas hasta los 150 días. Los resultados mostraron un excelente aprovechamiento ruminal de la planta de sorgo negro en etapas de crecimiento menores a 80 días, lo que permite recomendarlo para la alimentación del ganado lechero. 

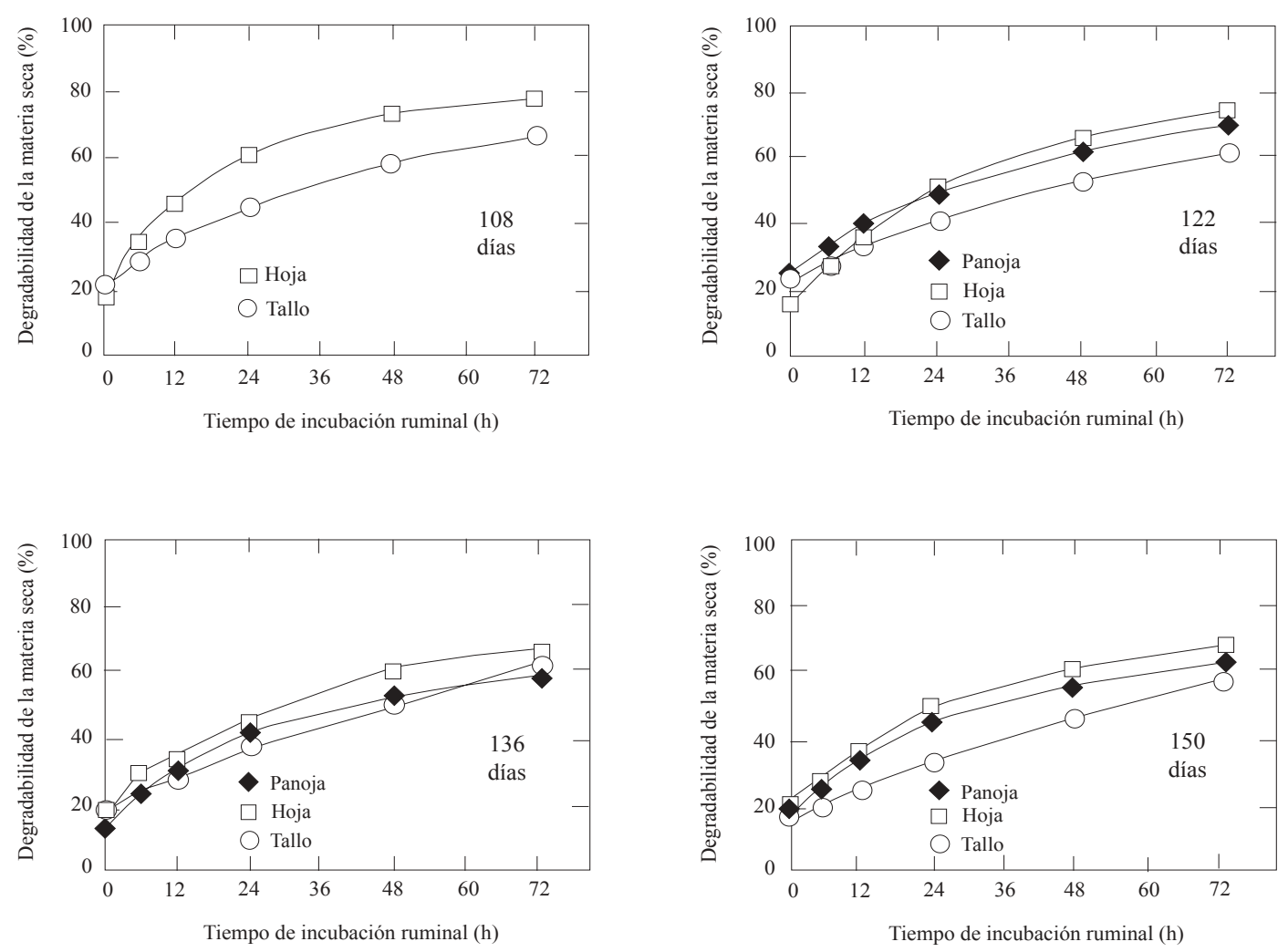

Figura 2. Degradabilidad ruminal de la materia seca de hojas y tallos de la planta de sorgo negro forrajero en estado de floración. Estación Experimental Alfredo Volio Mata, El Alto Ochomogo, Costa Rica 1999.

\section{LITERATURA CITADA}

ABARCA, S. 1988. Efecto de la suplementación con poró (Eritrina poeppigiana) y melaza sobre la producción de leche en vacas pastoreando Estrella Africana (Cynodon nlemfluensis). Tesis MSc. Centro Agronómico Tropical de Investigación y Enseñanza (CATIE), Turrialba, Costa Rica. 78 p.

AMADOR, A.; BOSCHINI, C. 2000. Calidad nutricional de la planta de sorgo negro forrajero (Sorghum almum) para alimentación animal. Agronomía Mesoamericana 11(2): 79-84.

BOSCHINI, C.; AMADOR, A. 2001. Degradabilidad ruminal de la planta de maíz en diferentes edades de crecimiento. Agronomía Mesoamericana 12(1): 89-93.

CORADO, L.H. 1991. Efecto de cuatro niveles de puliduras de arroz sobre la producción de leche de vacas en pastoreo suplementadas con follaje de poró (Eritrina poeppigiana) Tesis MSc. Centro Agronómico Tropical de Investigación y Enseñanza (CATIE) , Turrialba, Costa Rica. 84p.

CORRALES, J.C. 1986. Efecto de la densidad de siembra y la fertilización nitrogenada sobre la producción de biomasa y la calidad del sorgo negro forrajero (Sorghum almum). Tesis ingeniero agrónomo. Escuela de Zootecnia, Facultad de Agronomía, Universidad de Costa Rica, San José, Costa Rica. 67p.

HERNÁNDEZ, H.E. 1986. Fertilización nitrogenada y edad de corte para sorgo forrajero (Sorghum almum). Tesis ingeniero agrónomo. Facultad de agronomía, Universidad de Costa Rica. San José, Costa Rica. 102p.

HERNÁNDEZ, X. 1989. Comportamiento agronómico de tres forrajes de corte durante la época seca en Tilarán. Tesis Ingeniero Agrónomo. Facultad de Agronomía, Universidad de Costa Rica. San José, Costa Rica. 108 p.

HUGES, H.D. 1978. Forrajes. Traducción de Lorna J.L. 2da edición. México D.F. Compañía Editorial Continental $758 \mathrm{p}$.

JIMÉNEZ, C. 1995. Degradabilidad ruminal del sorgo negro forrajero (Sorghum almum). Tesis MSc. Programa de Posgrado en Ciencias Agrícolas y Recursos Naturales. Facultad de Agronomía. Universidad de Costa Rica. $61 p$.

MEDINA, P.J. 1988. Efecto de la suplementación con poró (Eritrina poeppigiana) y melaza sobre los parámetros de fermentación ruminal y la degradabilidad in situ del 
poró y pasto Estrella (Cynodon nlemfluensis). Tesis MSc. Centro Agronómico Tropical de Investigación y Enseñanza (CATIE), Turrialba, Costa Rica. 73p.

MURILLO, F. 1990. Efecto del nivel de fertilización y del intervalo de corte sobre la desaparición ruminal de la materia seca del pasto Estrella (Cynodon nlemfluensis (Vanderryst)). Tesis ingeniero agrónomo. Escuela de Ciencias Agrarias. Universidad Nacional. Heredia, Costa Rica. 112 p.

MORENO, M. 1991. Desaparición ruminal del pasto (Pennissetum purpureum var. King Grass) (Planta entera) con cuatro niveles de fertilización nitrogenada y tres intervalos de corte. Tesis ingeniero agrónomo. Escuela de Ciencias Agrarias. Universidad Nacional. Heredia, Costa Rica. 90p.

ORSKOV. E.R. 1984. Evaluation of residues and agroindustrial by-products using the nylon bag method. FAO-ILCA Publication. Roma, Italia. 128p.

ORSKOV. E.R.; Mc DONALD, I. 1979. The estimation of protein degradability in the rumen from incubation measurements weighted according to the rate of passage. Journal of Agricultural Science 92: 499-503.

OWEN, F.G.; MOLINE, W. J. 1970. Sorghum for forage; sorghum production and utilization. Wall and Ross. Westport, Va, USA. 320p.

VASQUEZ, A. 1982. Estudio detallado de los suelos de la Estación Experimental de Ganado Lechero El Alto. Escuela de Fitotecnia. Facultad de Agronomía. Universidad de Costa Rica. p. 36.

VILLEGAS, O. 1990. Producción y valor nutricional de sorgos forrajeros y sus ensilados a diferentes edades de cosecha. Tesis Ing. Agrónomo. Sede Regional de Guanacaste. Universidad de Costa Rica. Liberia. Costa Rica. 91p.

ZÚÑIGA, A.M. 1992. Efecto de la cáscara de banano sobre la degradabilidad de varios forrajes tropicales. Tesis Ing. agrónomo. Escuela de Zootecnia, Facultad de Agronomía. Universidad de Costa Rica, San José, Costa Rica. $64 p$. 